\title{
Rheumatology
}

\section{Multiple pathways of type 1 interferon production in lupus: the case for amlexanox.}

\begin{tabular}{|c|c|}
\hline Journal: & Rheumatology \\
\hline Manuscript ID & RHE-20-0856.R1 \\
\hline Manuscript Type: & Letter to the Editor (Other) \\
\hline $\begin{array}{r}\text { Date Submitted by the } \\
\text { Author: }\end{array}$ & $\mathrm{n} / \mathrm{a}$ \\
\hline Complete List of Authors: & $\begin{array}{l}\text { Todd, Ian; University of Nottingham, School of Life Sciences } \\
\text { Thomas, Rhema; University of Nottingham, School of Life Sciences } \\
\text { Watt, Baltina; University of Nottingham, School of Life Sciences } \\
\text { Sutherland, Lissa; University of Nottingham, School of Life Sciences } \\
\text { Afriyie-Asante, Afrakoma; University of Nottingham, School of Life } \\
\text { Sciences } \\
\text { Deb, Bishnu; University of Nottingham, School of Life Sciences } \\
\text { Joseph, Blessy; University of Nottingham, School of Life Sciences } \\
\text { Tighe, Paddy; University of Nottingham, School of Life Sciences } \\
\text { Lanyon, Peter; Nottingham University Hospitals NHS Trust, Queens } \\
\text { Medical Centre } \\
\text { Fairclough, Lucy; University of Nottingham, School of Life Sciences }\end{array}$ \\
\hline $\begin{array}{r}\text { Keywords }<\text { br }>\text { Please select a } \\
\text { minimum FIVE keywords from } \\
\text { the list provided. These } \\
\text { keywords will be used to } \\
\text { select reviewers for this } \\
\text { manuscript. The keywords in } \\
\text { the main text of your paper } \\
\text { do not need to match these } \\
\text { words.: }\end{array}$ & $\begin{array}{l}\text { Systematic lupus erythematosus and autoimmunity }<\text { RHEUMATIC } \\
\text { DISEASES, Cytokines and inflammatory mediators < BASIC \& CLINICAL } \\
\text { SCIENCES, Inflammation < BASIC \& CLINICAL SCIENCES, } \\
\text { Immunosuppressants < THERAPIES, Immunotherapy < THERAPIES }\end{array}$ \\
\hline
\end{tabular}




\section{Letter to the Editor}

Multiple pathways of type 1 interferon production in lupus: the case for amlexanox.

\section{Ian Todd ${ }^{1,3}$, Rhema E. Thomas ${ }^{1}$, Baltina D. Watt ${ }^{1}$, Lissa Sutherland ${ }^{1}$, Afrakoma Afriyie-Asante ${ }^{1}$, Bishnu Deb ${ }^{1}$, Blessy Joseph ${ }^{1}$, Patrick J. Tighe ${ }^{1,3}$, Peter Lanyon ${ }^{2,3}$, and Lucy C. Fairclough ${ }^{1,3}$}

${ }^{1}$ School of Life Sciences, The University of Nottingham, UK; ${ }^{2}$ Department of Rheumatology, Nottingham University Hospitals NHS Trust, Nottingham, UK; ${ }^{3}$ Nottingham Biomedical Research Centre (Musculoskeletal), Nottingham University Hospitals NHS Trust, Nottingham, UK.

Correspondence to: Lucy C. Fairclough, School of Life Sciences, The University of Nottingham, Life Sciences Building, University Park, Nottingham, NG7 2RD, UK.

Key message: Amlexanox inhibits multiple pathways of type-1 interferon production and may be therapeutically useful in lupus. 
Sir, Substantial evidence implicates type-1 interferons (IFN-1s) in the pathophysiology of systemic lupus erythematosus (SLE, lupus), and this has led to the development of several therapeutic strategies for lupus that target the IFN-1 pathways [1]. In particular, it has recently been reported that the therapeutic monoclonal antibody, anifrolumab, showed efficacy in meeting its primary endpoint in a phase III clinical trial in active lupus [2]. Anifrolumab targets the IFN-1 receptor and thereby inhibits the activity of all species of IFN$1(\alpha, \beta$ and $\omega)$. Overall, anifrolumab has shown greater promise in lupus than the therapeutic monoclonal antibodies that directly target IFN- $\alpha$ only (i.e. sifalimumab, rontalizumab) [1]. This is not surprising, as although many studies have concentrated on the role of IFN- $\alpha$ derived from plasmacytoid dendritic cells in lupus, it is now apparent that IFN- $\beta$ and IFN- $\omega$ are also important in the pathophysiology [3]. With regard to the signalling pathways that are triggered in lupus to generate IFN-1 production, emphasis has been placed on the role of the DNA sensor TLR9 [4]. However, there is increasing evidence for the involvement of the RNA sensor TLR3, RNA sensors such as RIG-1/MDA-5 that stimulate MAVS, and the DNA sensors such as cGAS that stimulate STING [4-6]: all of these three pathways act via the signalling molecule TBK-1. Indeed, the type 1 IFN gene signature in peripheral blood mononuclear cells of childhood-onset lupus patients can be down-regulated by the TBK-1 inhibitor BX795 [5]. These and other findings have raised the potential of TBK-1 inhibitors as therapeutic agents for lupus [5].

Amlexanox is used for the topical treatment of aphthous ulcers and has been used to a limited degree in allergic asthma. We previously highlighted amlexanox from screening 1300 drugs for inhibitory effects on inflammatory signalling pathways [7] and it

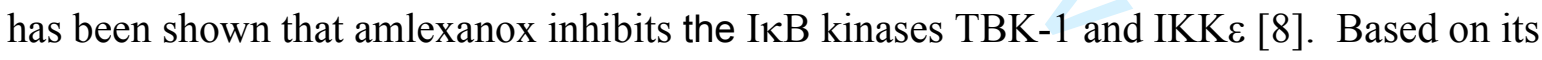
inhibition of TBK-1 and IKKe, amlexanox was recently used systemically in a randomized, double-blind, placebo-controlled clinical study in obese patients with type II diabetes and non-alcoholic fatty liver disease; it was shown to improve glucose control in a subset of patients with an inflammatory profile [9]. Only low-grade adverse side-effects were seen during the study. Importantly, therefore, this study demonstrated the clinical efficacy and safety of amlexanox when used systemically in patients [9].

Numerous subsequent studies in vitro and in animal models have demonstrated the effectiveness of amlexanox as a TBK-1 inhibitor (e.g. [10]) in a variety of pathological settings although, to our knowledge, no studies of amlexanox in relation to lupus have been reported to date. However, the potential of amlexanox in lupus is strongly supported by all of 
these other studies. It is important, therefore, to be clear that amlexanox inhibits all three of the pathways involving TBK-1 that may contribute to the production of IFN-1s in lupus. (Most other studies have examined the inhibitory effects of amlexanox on just one or two of these pathways: e.g. Raicevic et al. demonstrated that amlexanox inhibits IFN- $\beta$ production by mesenchymal stem cells stimulated via the RIG-1/MDA-5 pathway [10].)

In this regard, we now present evidence that amlexanox downregulates all three pathways of TBK-1 activation involving TLR-3, RIG-1/MDA-5/MAVS or cGAS/STING in the same cell type. For this we employed the lung epithelial carcinoma A549-Dual ${ }^{\mathrm{TM}}$ reporter cell line (Invivogen) that expresses Lucia luciferase under the control of IFNstimulated response elements. Thus, induction of luciferase expression acts as a surrogate for IFN-1 production and is detected by the action of luciferase on the QUANTI-Luc ${ }^{\text {TM }}$ detection $^{-1}$ reagent (Invivogen). The cells were cultured for $16 \mathrm{~h}$ at $5 \times 10^{4} /$ well in 96 -well plates either without ligands, or with one of the following: $50 \mu \mathrm{g} / \mathrm{mL}$ Poly I:C (TLR-3 ligand), $0.1 \mu \mathrm{g} / \mathrm{mL}$

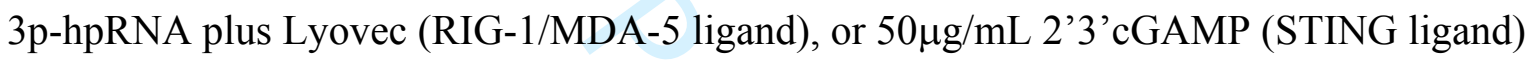
(all from Invivogen). The cells were cultured with or without $1 \mu \mathrm{M}$ amlexanox (Tocris Bioscience). All the cultures contained dimethyl sulphoxide (1\%) as a control as this was the solvent for amlexanox. After $16 \mathrm{~h}, 20 \mu \mathrm{l}$ aliquots of the culture supernatants were added to $50 \mu 1$ aliquots of QUANTI-Luc ${ }^{\mathrm{TM}}$ and the luminescence generated was immediately measured.

The results shown in figure 1 for three to six independent experiments are expressed as ratios of the luminescence values of supernatants from stimulated cells divided by the luminescence values of supernatants from cells cultured without ligands or amlexanox. These results show that amlexanox significantly inhibited the stimulation of the A549-Dual ${ }^{\mathrm{TM}}$ cells by all three ligands/pathways. Amlexanox did not induce death of the cells as determined by trypan blue exclusion: cell death was only $2-3 \%$ on average following culture without or with amlexanox $(\mathrm{P}=0.4)$.

Amlexanox did not affect the viability of the cells (data not shown).

These findings of the effects of amlexanox using the A549-Dual ${ }^{\mathrm{TM}}$ reporter cell line $\underline{\text { should be confirmed in experiments with human peripheral blood mononuclear cells }}$ (PBMCs), using synthetic ligands or sera from SLE patients to stimulate IFN-1 production. Although these experiments are beyond the scope of this letter, we have preliminary data showing that amlexanox inhibits IFN- $\beta$ production by Poly I:C-stimulated PBMCs (data not shown). However, our current data does show Our data therefore shows-that amlexanox 
inhibits all three pathways leading to TBK-1 activation and type 1 IFN production in response to particular forms of DNA or RNA ligands. We propose that this finding, together with the previous demonstration of the efficacy and safety of amlexanox administered systemically in a clinical trial in type II diabetes and non-alchoholic fatty liver disease [9], provides support for amlexanox to be considered for trials as a novel therapeutic agent in lupus.

\section{Acknowledgements}

The authors thank Colin Nicholson for technical assistance.

Funding: This work was supported by grants from LUPUS UK and The Nottingham Biomedical Research Centre (Musculoskeletal).

Disclosure statement: The authors have declared no conflicts of interest.

\section{References}

1 Klavdianou K, Lazarini A, Fanouriakis A. Targeted Biologic Therapy for Systemic Lupus Erythematosus: Emerging Pathways and Drug Pipeline. BioDrugs 2020.

2 Morand EF, Furie R, Tanaka Y, et al. Trial of Anifrolumab in Active Systemic Lupus Erythematosus. N Engl J Med 2020;382(3):211-21.

3 Catalina MD, Bachali P, Geraci NS, Grammer AC, Lipsky PE. Gene expression analysis delineates the potential roles of multiple interferons in systemic lupus erythematosus. Commun Biol 2019;2:140.

4 Klonowska-Szymczyk A, Wolska A, Robak T, Cebula-Obrzut B, Smolewski P, Robak E. Expression of toll-like receptors 3, 7, and 9 in peripheral blood mononuclear cells from patients with systemic lupus erythematosus. Mediators of inflammation 2014;2014:381418. 5 Wahadat MJ, Bodewes ILA, Maria NI, et al. Type I IFN signature in childhood-onset systemic lupus erythematosus: a conspiracy of DNA- and RNA-sensing receptors? Arthritis research \& therapy 2018;20(1):4.

6 Wang J, Dai M, Cui Y, et al. Association of Abnormal Elevations in IFIT3 With Overactive Cyclic GMP-AMP Synthase/Stimulator of Interferon Genes Signaling in Human Systemic Lupus Erythematosus Monocytes. Arthritis \& rheumatology 2018;70(12):2036-45. 7 Todd I, Negm OH, Reps J, et al. A signalome screening approach in the autoinflammatory disease TNF receptor associated periodic syndrome (TRAPS) highlights the anti-inflammatory properties of drugs for repurposing. Pharmacological research 2017;125(Pt B):188-200.

8 Reilly SM, Chiang SH, Decker SJ, et al. An inhibitor of the protein kinases TBK1 and IKK-varepsilon improves obesity-related metabolic dysfunctions in mice. Nat Med 2013;19(3):313-21.

9 Oral EA, Reilly SM, Gomez AV, et al. Inhibition of IKKvarepsilon and TBK1 Improves Glucose Control in a Subset of Patients with Type 2 Diabetes. Cell Metab 2017;26(1):157-70 e7. 
10 Raicevic $\mathrm{G}$, Najar $\mathrm{M}$, Busser $\mathrm{H}$, et al. Comparison and immunobiological characterization of retinoic acid inducible gene-l-like receptor expression in mesenchymal stromal cells. Scientific reports 2017;7(1):2896.

\section{Figure legend}

FIG 1. Amlexanox suppresses the activation of multiple signalling pathways involving

TBK-1. A549-Dual ${ }^{\mathrm{TM}}$ cells were cultured for $16 \mathrm{~h}$ with the indicated ligands $(50 \mu \mathrm{g} / \mathrm{mL}$ Poly $\mathrm{I}: \mathrm{C}, 0.1 \mu \mathrm{g} / \mathrm{mL} 3 \mathrm{p}-\mathrm{hpRNA}$ plus Lyovec, or $50 \mu \mathrm{g} / \mathrm{mL}$ 2'3'cGAMP), and with or without $1 \mu \mathrm{M}$ amlexanox. The culture supernatants were then tested for secreted luciferase by addition to QUANTI-Luc ${ }^{\mathrm{TM}}$ detection reagent. The results are presented as luminescence ratios of $\underline{\text { luciferase activity of ligand-stimulated cultures (without or with amlexanox) divided by the }}$ luciferase activity of non-stimulated cultures. Paired t-test was used to compare the readings in the absence or presence of amlexanox ( $p<0.05$ considered significant). 


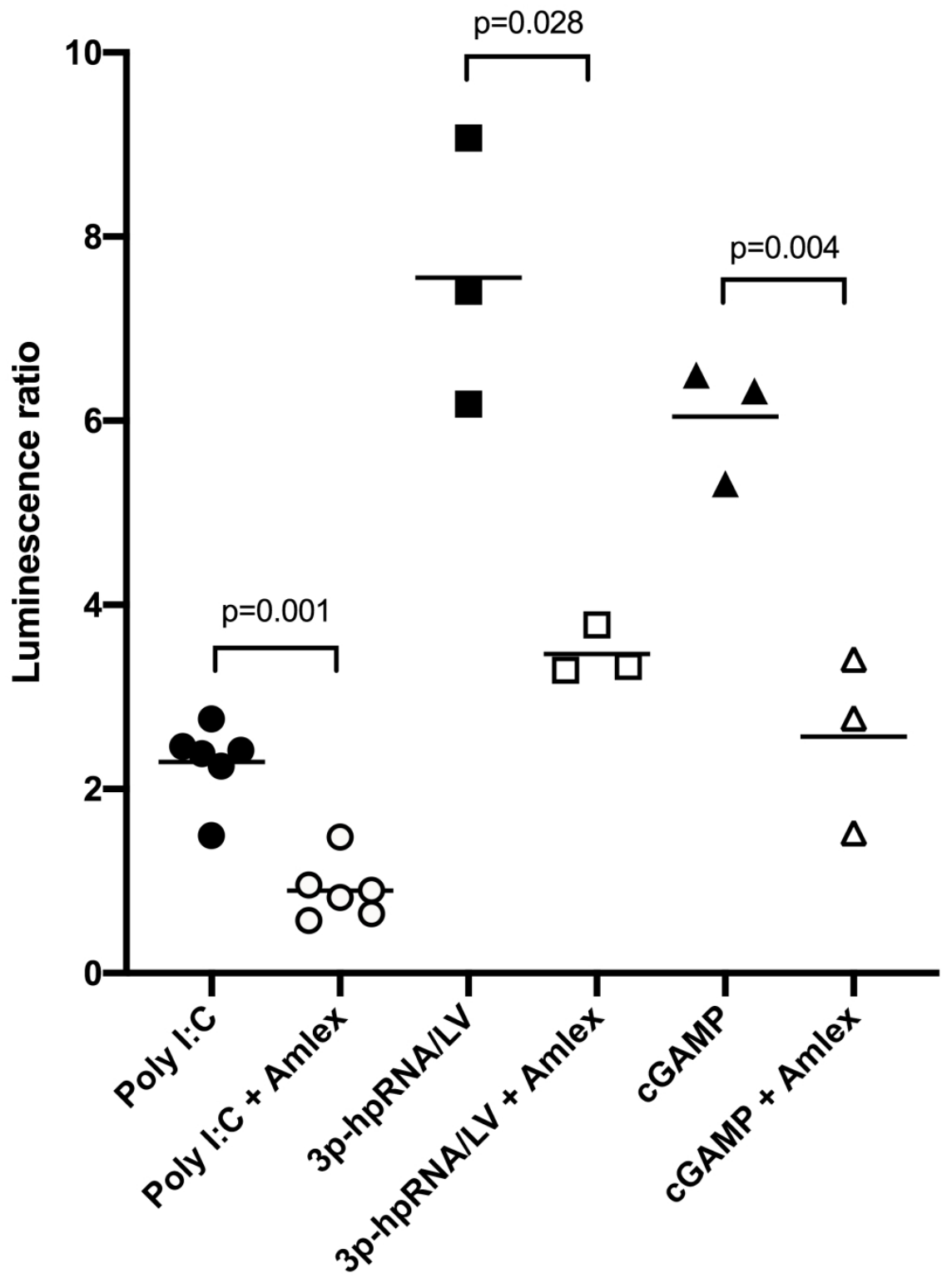

FIG 1. A549-DualTM cells were cultured for $16 \mathrm{~h}$ with the indicated ligands $(50 \square \mathrm{g} / \mathrm{mL}$ Poly I:C, $0.1 \square \mathrm{g} / \mathrm{mL}$ $3 p-h p R N A$ plus Lyovec, or $50 \square \mathrm{g} / \mathrm{mL} 2^{\prime} 3^{\prime} \mathrm{cGAMP}$ ), and with or without $1 \square \mathrm{M}$ amlexanox. The culture supernatants were then tested for secreted luciferase by addition to QUANTI-LucTM detection reagent. The results are presented as luminescence ratios of luciferase activity of ligand-stimulated cultures (without or with amlexanox) divided by the luciferase activity of non-stimulated cultures. Paired t-test was used to compare the readings in the absence or presence of amlexanox $(p<0.05$ considered significant).

$109 \times 146 \mathrm{~mm}(600 \times 600 \mathrm{DPI})$ 